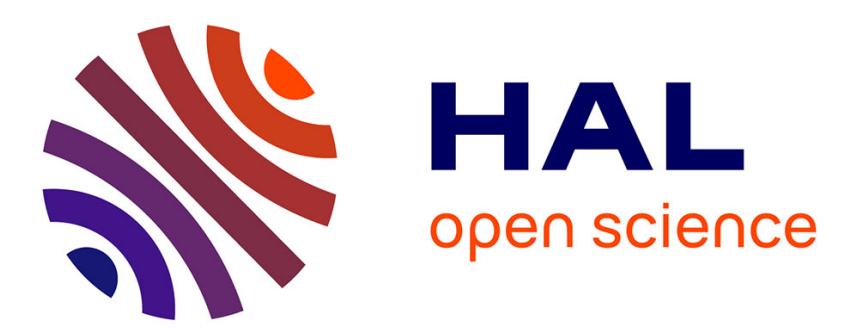

\title{
Characterization of Radiating Sources in the Near Field Using EMTR Technique: A Parametric Study
}

Sassia Hedia, Bessem Zitouna, Jaleleddine Ben Hadj Slama, Lionel Pichon

\section{To cite this version:}

Sassia Hedia, Bessem Zitouna, Jaleleddine Ben Hadj Slama, Lionel Pichon. Characterization of Radiating Sources in the Near Field Using EMTR Technique: A Parametric Study. 6th IEEE International Energy Conference, 2020, Gammarth, Tunisia. 10.1109/ENERGYCon48941.2020.9236581. hal-03420340

\section{HAL Id: hal-03420340 https://hal.science/hal-03420340}

Submitted on 9 Nov 2021

HAL is a multi-disciplinary open access archive for the deposit and dissemination of scientific research documents, whether they are published or not. The documents may come from teaching and research institutions in France or abroad, or from public or private research centers.
L'archive ouverte pluridisciplinaire HAL, est destinée au dépôt et à la diffusion de documents scientifiques de niveau recherche, publiés ou non, émanant des établissements d'enseignement et de recherche français ou étrangers, des laboratoires publics ou privés. 


\section{Characterization of Radiating Sources in the Near Field Using EMTR Technique : A Parametric Study}

\author{
Sassia HEDIA \\ ${ }^{1}$ Université de Sousse, Ecole \\ Nationale d'Ingénieurs de Sousse, \\ LATIS- Laboratory of Advanced \\ Technology and Intelligent \\ Systems, 4023, Sousse, Tunisie. \\ ${ }^{2}$ Université Paris-Saclay, \\ CentraleSupélec, CNRS, \\ Laboratoire de Génie Electrique \\ et Electronique de Paris, 91192, \\ Gif-sur-Yvette, France. \\ sassia.hedia@supelec.fr
}

\author{
Bessem ZITOUNA \\ ${ }^{1}$ Université de Sousse, Ecole \\ Nationale d'Ingénieurs de \\ Sousse, LATIS- Laboratory of \\ Advanced Technology and \\ Intelligent Systems, 4023, \\ Sousse, Tunisie. \\ bessem.zitouna@yahoo.fr
}

\author{
Jaleleddine BEN HADJ \\ SLAMA \\ ${ }^{1}$ Université de Sousse, Ecole \\ Nationale d'Ingénieurs de Sousse, \\ LATIS- Laboratory of Advanced \\ Technology and Intelligent \\ Systems, 4023, Sousse, Tunisie. \\ bhslama@yahoo.fr
}

\author{
Lionel PICHON \\ ${ }^{2}$ Université Paris-Saclay, \\ CentraleSupélec, CNRS, \\ Laboratoire de Génie Electrique \\ et Electronique de Paris, 91192 , \\ Gif-sur-Yvette, France. Sorbonne \\ Université, CNRS, Laboratoire de \\ Génie Electrique et Electronique \\ de Paris, 75252, Paris, France. \\ Lionel.Pichon@supelec.fr
}

\begin{abstract}
The aim of this study is the investigation of the effects of parameters involved in the characterization of radiating sources in the near field (NF). The main issue is to deal with structures of power electronic systems that are generally excited by non-sinusoidal signals. The identification is based on the electromagnetic time reversal (EMTR) technique. Indeed, the electromagnetic field was synthesized analytically using expressions of equivalent dipoles radiation in time domain (TD). The effects of several parameters on sources characterization, including the height of measurement, time reversal mirror (MRT), potential sources network, and the measuring probe were evaluated. Several simulation tests have been applied to this set of parameters to determine their simultaneous effect on the identification of radiated emissions of a power electronic structure. Based on this parametric study, a compromise between the accuracy of the obtained equivalent model and an acceptable computing time is determined and demonstrated to provide an efficient reconstruction.
\end{abstract}

Keywords-Electromagnetic time reversal, radiated emissions, near field scanning technique, power electronic systems.

\section{INTRODUCTION}

In the literature, several research works have been presented in order to study electromagnetic compatibility (EMC) in power and energy systems [1-2]. Indeed, EMC has been established on the sensitivity of electronic circuits, usually operating in close proximity to powerful sources of electromagnetic (EM) interferences [3-4]. Therefore, the identification of radiating sources in the near field (NF) has been of great interest [2-5].

Recently, EM inverse method based on electromagnetic time reversal (EMTR) has been presented as an efficient method for characterizing radiating sources in the NF [6-7]. In [8], EMTRbased method has been applied for non-sinusoidal radiation signals. These results have been carried out using simulation results and experimental measurements. The EMTR-based method is based on a full time domain (TD) analysis. For validation purposes, in [9], a comparative study has been carried out between the proposed method and a conventional genetic algorithm based method.

Indeed, EM inverse method is based on the identification of a set of magnetic equivalent dipoles in the near field region. Several parameters affect the quality of the identification of a representative radiation model. These variables limit the efficiency and the convergence of any method [3] [5]. Indeed, on the one hand, parameters such as height of measurements, scanning resolution and the source characteristics (radius, excitation, etc.) influence the resulting radiation magnitude. On the other hand, the proposed EMTR-based method mainly depends on some of these variables such as the TRM, potential sources network and the reference radiation strength.

This paper aims to investigate the influence of a set of parameters as variables to be used within the EMTR-based method to characterize radiating sources. Simulations have been performed in order to obtain a compromise between results accuracy and the computing time in case of a power electronic structure. Within this context, the paper is organized as follows: Section II states theoretical formulations of EM inverse method based on EMTR. In Section III, a sensitivity study is applied to a multisource structure carrying transient excitations. Simulation results based on the magnetic field and the focusing quality are presented to assess the obtained characterization. A discussion is provided in Section IV. Conclusions are presented in Section V.

\section{THEORETICAL INVESTIGATIONS}

\section{A. Electromagnetic Time Reversal}

Time reversal (TR) has been presented in the literature as an interesting signal processing technique with a potential various applications and fields of engineering [10-12]. In particular, TR has been applied in the field of EMC and power electronic systems [11]. For instance, EMTR has been used to locate sources of EM radiation in transmission lines and lightning [11- 
12]. An application of EMTR technique for sources characterization in an EMC context has been presented in [8].

Fig. 1. depicts the main steps of an EMTR experiment. This technique is based on the synchronized recording of magnetic field waveforms emitted by a device under test (DUT), on the time reversal mirror (TRM). Then, these records are time reversed and back propagated in the location domain using analytical simulations. In the real source location, backpropagated field waveforms add up constructively to reach a maximum at the focusing time. The peak value of the obtained sum depends on several parameters, mainly the test configuration and sources characteristics.

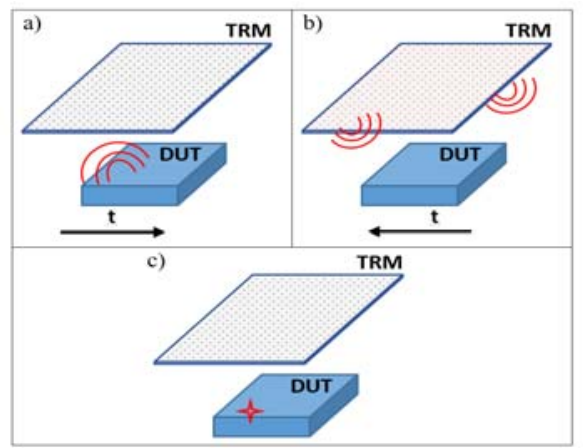

Fig. 1. Time Reversal : a) A source emits a transient radiation in the medium and recording of radiation waveforms at the TRM. b) Time reversing of EM field and back-prpagation. $c$ ) Focusing and source identification.

\section{B. Proposed Method}

In order to characterize EM emissions using NF scanning technique, an adequate equivalent model has to be determined. Indeed, based on the inverse problem resolution, the EM radiation behaviour of a DUT can be represented as a set of elemental dipoles radiating the same emissions. Fig.2. illustrates the principle of the proposed EM modelling. Each dipole is defined, as in [13], by an array of six parameters that characterize a radiating magnetic source, such as the centre coordinates $\left(X_{d}, Y_{d}, Z_{d}\right)$, the angles of orientation of the magnetic moment $(\varphi, \theta)$, and the excitation signal.

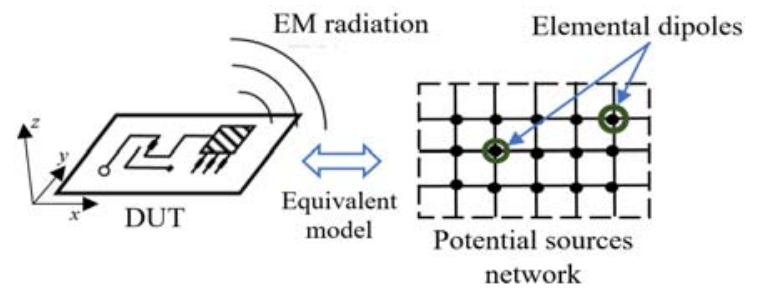

Fig. 2. Equivalent dipole model.

The source location identification has been performed using an EM inverse method is based on EMTR, as explained in [6] and [8]. A potential sources network is defined as the calculation domain (mesh elements). Hence, the proposed method is based on the determination of the potential source that has the maximum focusing magnitude. Indeed, this virtual network is a set of point-like sources that can be a real radiating source (a potential source). Thus, we first assign for each element of the network a position, an orientation and a pulse excitation. Thereafter, the transfer matrix is calculated, which allows obtaining focusing signals as detailed in [8]. Once the most radiating source has been determined among the virtual network, using the focusing criteria, parameters of the identified dipole are determined. Then, the obtained equivalent dipoles number is iterated. Therefore, the sum of the contributions from each identified dipole is updated (estimated map). In fact, due to temporal and spatial focusing property of EMTR, the resulting focus occurs at the source location at a particular time step, depending on the TRM and the potential sources network. Hence, a parametric study is very interesting in order to optimize the identification process by choosing an adequate configuration for the characterization of radiated emissions.

\section{SENSITIVITY ANALYSIS}

The following parametric study is carried out using the EM inverse method based on EMTR. Analytical equations of magnetic dipoles radiation have been implemented in TD as in [8], in a simulated environment (MATLAB). Therefore, the present work does not consider measurement errors. The influence of parameters involved in EM radiation characterization such as configuration variables is evaluated in respect of identification results. For the scope of this paper, only $H_{z}$ component is presented. Identified dipoles are used in order to obtain estimated maps. The relative error is quantified between simulated and estimated maps at a fixed time step.

In addition, in this work, the sensitivity analysis is applied to a general structure. Generally, in power electronic systems, a representative radiation model is based on a limited number of different equivalent dipoles (relatively five equivalent dipoles) [5]. This set of elementary sources mainly represents electronic components such as inductances, transistors, diodes, etc. Therefore, in this parametric study, for simplification purposes, we arbitrary place five horizontal current loops with radius $r=$ $5 \mathrm{~mm}$, on a scanning area of $(21 \mathrm{~cm} \times 21 \mathrm{~cm})$. Table I presents initial simulated model parameters. Moments corresponding to real sources are depicted in Fig. 3.
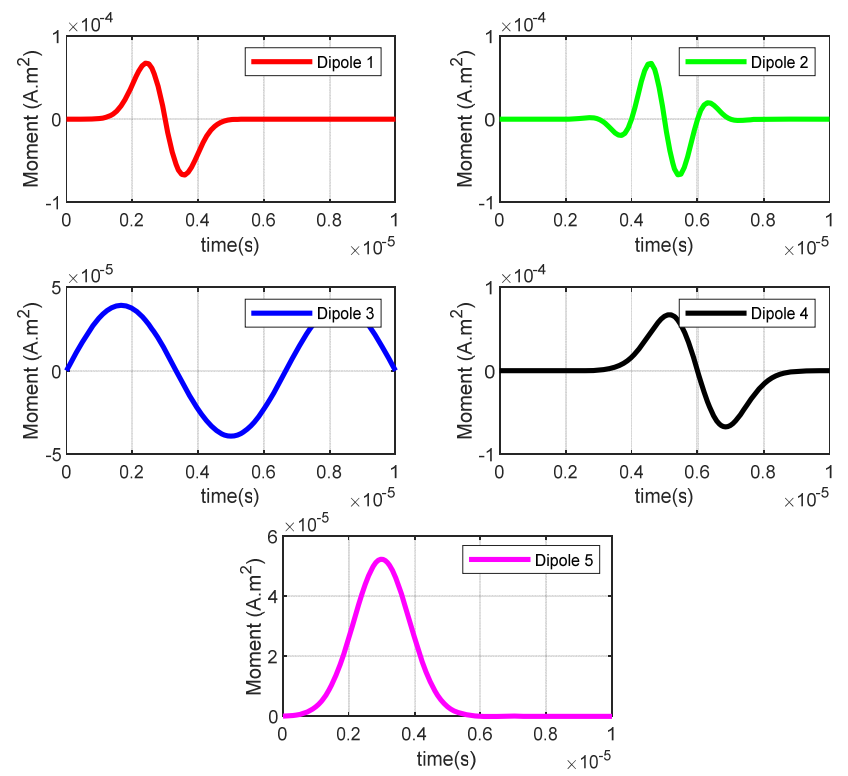

Fig. 3. Initial moment signals. 
TABLE I. SIMULATED MODEL PARAMETERS

\begin{tabular}{|c|c|ccccc|c|}
\hline $\begin{array}{c}\text { Source } \\
\text { number }\end{array}$ & $\begin{array}{c}\text { Max Moment } \\
\left(\mathbf{A . m ^ { 2 } )}\right.\end{array}$ & $\begin{array}{r}{\left[\boldsymbol{X}_{\boldsymbol{d}} \boldsymbol{Y}_{\boldsymbol{d}}\right.} \\
(\mathbf{m m})\end{array}$ & $\left.\boldsymbol{Z}_{\boldsymbol{d}}\right]$ & $\begin{array}{c}\text { Orientation } \\
(\text { rad })\end{array}$ \\
\hline$\# 1$ & $2.4 \times e^{-6}$ & 0 & $;$ & 0 & $;$ & 0 & 0 \\
\hline$\# 2$ & $4.5 \times e^{-6}$ & -6 & $;$ & -6 & $;$ & 0 & 0 \\
\hline$\# 3$ & $5.2 \times e^{-6}$ & -4.5 & $;$ & 7.5 & $; 0$ & 0 & 0 \\
\hline$\# 4$ & $4.1 \times e^{-6}$ & -7.5 & $;$ & $3 \quad ; 0$ & 0 \\
\hline$\# 5$ & $3 \times e^{-6}$ & -1.5 & $;$ & $7.5 ; 0$ & 0 \\
\hline
\end{tabular}

\section{A. Height of measurements}

In EMC studies, while measuring radiated emissions, the distance of scan is a very important parameter. Indeed, in the literature, researchers have investigated the NF scanning technique in order to obtain an accurate representation of the DUT [2-9]. NF measurement has interesting advantages in terms of cost, accuracy, and application range. Moreover, the NF scan is less dependent on test conditions. Indeed, the higher the scanning distance, the field approximations are more likely to be invalid. A closer scanning height leads to a more efficient modelling of the DUT. In addition, the EM field strength dramatically decreases with the increase of scanning distance. Therefore, in this work, the height of measurement is limited by the near field definition. This means that the distance between the source and the scan surface is inferior to $\frac{\lambda}{2 \pi}$, where $\lambda$ is the wavelength defined by $\lambda=\frac{c}{f}$, where $f$ is the operating frequency. Indeed, in power electronics such as converters and switching devices, systems are operating in a frequency range from few $\mathrm{kHz}$ up to tens of megahertz. In this context, a high scanning distance leads to a low Signal to Noise Ratio (SNR) and thus a significant measurement errors that may affect the characterization accuracy. Contrariwise, a low height can creates coupling effects between the measuring probe and the DUT. The latter will also affects the field distributions above the circuit and provides erroneous results.

In order to demonstrate previous foundations, we evaluate the influence of the height of measurements on the obtained field maps and focusing using the defined multisource structure. Hence, we only vary the height of scan, as in Table II. General configurations parameters are the same for all tests. TRM and virtual sources network sizes are both set to $(15 \times 15)$.

TABLE II. INFLUENCE OF HEIGHT OF MEASUREMENTS

\begin{tabular}{|c|c|c|c|c|}
\hline \multicolumn{5}{|c|}{ Configuration 1 (in m) } \\
\hline Test 1 & Test2 & Test 3 & Test 4 & Test 5 \\
\hline 0.005 & 0.0085 & 0.01 & 0.02 & 0.05 \\
\hline
\end{tabular}

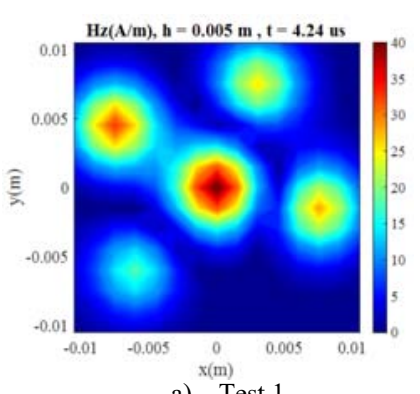

a) Test 1

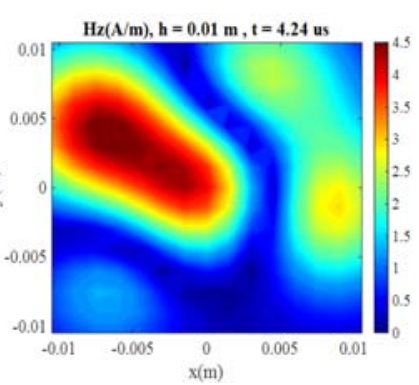

b) Test 3
Fig. 4. Influence of height of measurement on radiated field maps.
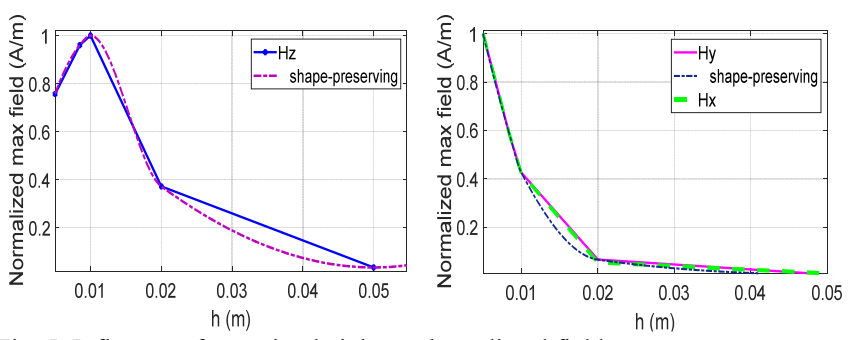

Fig. 5. Influence of scanning height on the radiated field.

Figs. 4 and 5 illustrate the impact of the increase of the scanning distance on the magnetic field strength. Indeed, radiated maps at time step $t=4.24$ us show that the field quality deteriorates when the height increases. Fig. 6 shows the influence of the height on focusing magnitude. An increase in the scanning distance dramatically reduce the spatial focusing.

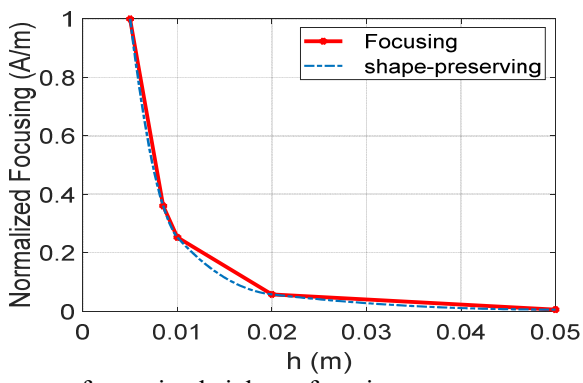

Fig. 6. Influence of scanning height on focusing.

\section{B. Time Reversal Mirror}

Another important parameter with regard to the characterization of radiating sources in the NF is the size of the scanning area, also known as time reversal mirror in the EMTRbased method. In fact, the scan surface is discretized with a spatial sampling accurately chosen. Indeed, if any significant EM field area is not covered, certain information will be lost and the equivalent model obtained from these data would have noticeable errors. It is well established that magnetic NF maps depend on routing, positioning and characteristics of electronic components which radiations may extend outward to the perimeter of the DUT. The latter implies that, in order to better represent emissions, the scanning area has to cover at least the area of the DUT, and preferably extend beyond it. Ideally, the scanning area should extend until the measured EM fields on the edges of the surface reach the minimum measurable level (noise) of the device. Nevertheless, in practice, it is not necessary to scan too widely to collect sufficient NF data. It is worth noting that when the scans are performed at a higher distance, a same scanning area covers less information volume. In order to obtain an adequate representation of NF radiation maps, the higher the scanning distance, the wider scanning area.

Actually, the TRM is a network of measuring points (transducers) placed above the board of a DUT and defined by the scanning resolution. For instance, using a low TRM size, the obtained equivalent model might be erroneous. However, by using a dense TRM, a significant calculation time is required and measurements errors will be accumulated. Depending on 
the density of the DUT circuit, the increase of the number of radiating sources leads to an increase of the number of measuring points. Indeed, each identified source (defined as in II.B) requires the resolution a system of six equations corresponding to each parameter of the equivalent dipole. Therefore, the size of the TRM is defined as:

$N_{T R M}=6 \times k \times N_{I d S r}$

where $N_{T R M}=N_{x \text {-axis }} \times N_{y \text {-axis }}$ is the different scanning points number, $N_{I d S r}$ is the number of identified sources and $1<k$ is a multiplying factor that guarantees that sources are independent and do not reproduce any correlation.

The influence of the number of TRM elements is discussed with respect to the magnetic field and focusing results. Indeed, the EM inverse method based on EMTR is established on the use of two-dimensional TRM. The choice is related to the NF scanning technique in which it is recommended to scan the entire circuit surface. Hence, the scanning area has to be planar. The scan is performed over the DUT with variable scanning resolutions therefore different TRM sizes. Tests configuration are presented in Table III. The height of measurement is set at $h=0.005 \mathrm{~m}$ and the virtual sources network is $(15 \times 15)$.

TABLE III. INFLUENCE OF NUMBER OF TRM ELEMENTS

\begin{tabular}{|l|c|c|c|c|c|}
\hline \multirow{2}{*}{ Test } & \multicolumn{5}{|c|}{ Configuration 2 } \\
\cline { 2 - 6 } & $\boldsymbol{k}$ & $\begin{array}{c}\text { Scanning } \\
\text { points }\end{array}$ & $\begin{array}{c}\text { Scanning } \\
\text { resolution (m) }\end{array}$ & $\begin{array}{c}\text { Relative } \\
\text { error (\%) }\end{array}$ & $\begin{array}{c}\text { Computing } \\
\text { time (s) }\end{array}$ \\
\hline$\# 1$ & 1.2 & $(6 \times 6)$ & 0.0042 & 3.69 & 16.47 \\
\hline$\# 2$ & 7.5 & $(15 \times 15)$ & 0.0015 & 1.64 & 37.22 \\
\hline$\# 3$ & 61.6 & $(43 \times 43)$ & 0.0005 & 0.22 & 166.11 \\
\hline
\end{tabular}
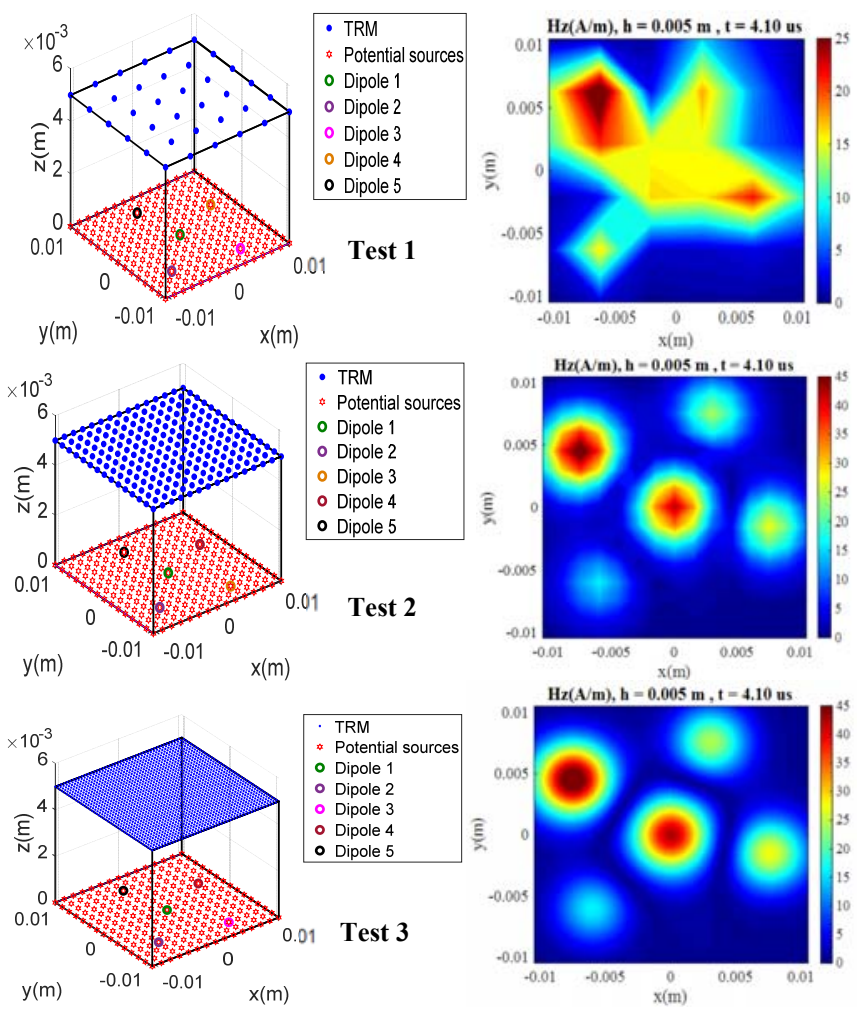

Fig. 7. Influence of number of TRM elements.
As shown in Fig. 7 and in Table III, the field quality is enhanced when the TRM size increases. This also implies that when a scanning area is large enough, further increasing its size does not significantly improve the accuracy of the equivalent model, such as in Tests 2 and 3. However, it is worth noting that for the different configuration tests, radiating sources characterization is always efficient, Fig 7 . Thereafter, the impact on focusing magnitude is investigated by considering it as a function of the size of TRM for each identified dipole. The magnitude of the maximum focusing peak tends to increase when the number of time reversal mirror's elements grows, as shown in Fig. 8.

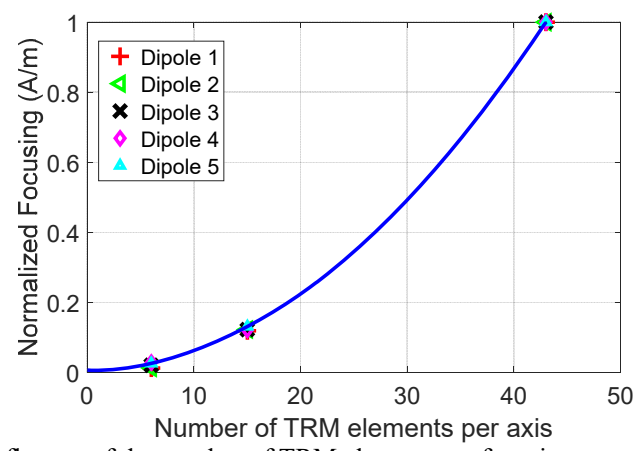

Fig. 8. Influence of the number of TRM elements on focusing.

\section{Potential sources Network}

Based on EMTR theory, potential sources network is deeply related to focusing phase. Practically speaking, we often want to focus an EM wave on one antenna of this array. Indeed, the identified dipole corresponds to the potential source that has the highest focusing magnitude at each iteration. It is obvious that elements of the potential sources network has a significant impact on the accuracy and the efficiency of the proposed method. In fact, a limited number of potential sources leads to erroneous equivalent model and therefore we don't obtain a good estimation of the position of radiating sources. Contrariwise, using a significant size of the virtual network, the identification will take a considerable calculation time.

In order to evaluate the influence of virtual sources network density, we have applied the EMTR-based method for different configurations in which the size of this network is variable. Indeed, the proposed identification method is based on the criteria of maximum magnitude of focusing as in [8]. In this subsection, the height of measurement is $h=0.005 \mathrm{~m}$. The size of the TRM is set at $(15 \times 15)$ scanning points.

TABLE IV. INFLUENCE OF POTENTIAL SOURCES NETWORK

\begin{tabular}{|c|c|c|c|c|}
\hline \multirow{2}{*}{ Test } & \multicolumn{4}{|c|}{ Configuration 3 } \\
\cline { 2 - 5 } & $\begin{array}{c}\text { Potential } \\
\text { sources network }\end{array}$ & $\begin{array}{c}\text { Scanning } \\
\text { resolution (m) }\end{array}$ & $\begin{array}{c}\text { Relative } \\
\text { error (\%) }\end{array}$ & $\begin{array}{c}\text { Computing } \\
\text { time (s) }\end{array}$ \\
\hline Test 1 & $(6 \times 6)$ & 0.0042 & 7.53 & 17.89 \\
\hline Test 2 & $(15 \times 15)$ & 0.0015 & 1.64 & 37.22 \\
\hline Test 3 & $(43 \times 43)$ & 0.0005 & 1.82 & 216.63 \\
\hline
\end{tabular}

Looking at Table IV, Figs. 9 and 10, we observe the influence of the number of potential sources network on the characterization of radiating sources. For instance, in Test 1, 
identified dipoles parameters give an erroneous estimation, Fig.9. However, if we increase the virtual sources network size, as in Test 2, an accurate identification is obtained, Fig.10. Therefore, it is obvious that the size of potential sources network has to be least the same as the TRM. Spatial focusing has been enhanced and the relative error between reference and estimated radiation maps has decreased, Table IV. Obtained model parameters in case of tests 1 and 2 are presented in Table V.
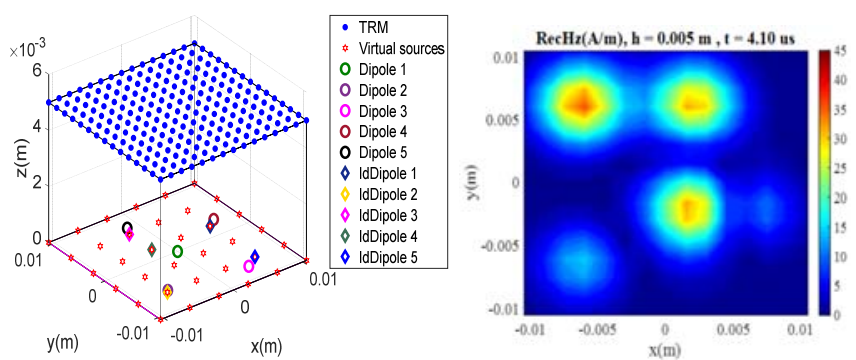

Fig. 9. Influence of potential sources network on the characterization: Test 1.
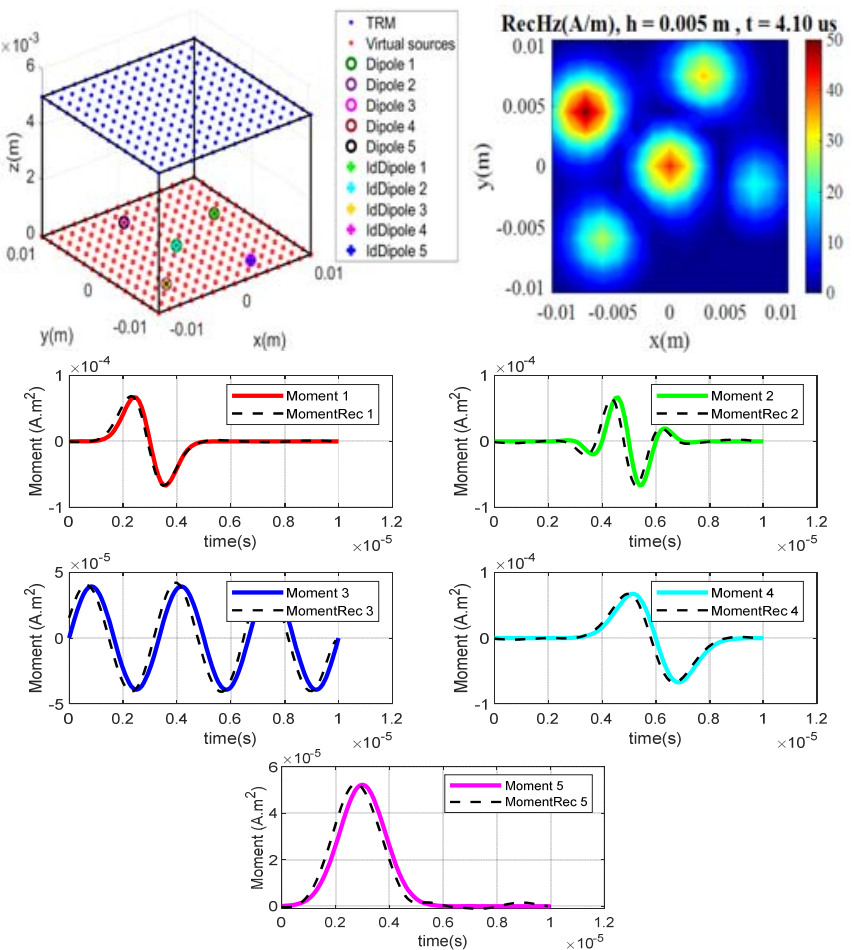

Fig. 10. Influence of potential sources network on the characterization: Test 2.

TABLE V. MODEL PARAMETERS OBTAINED BY THE PROPOSED METHOD

\begin{tabular}{|c|c|c|c|c|c|c|}
\hline & \multicolumn{5}{|c|}{ Test 1 } & \multicolumn{4}{|c|}{ Test 2 } \\
\cline { 2 - 8 } & $\begin{array}{c}M . \boldsymbol{e}^{-\mathbf{6}} \\
\left(\mathbf{A . m ^ { 2 } )}\right.\end{array}$ & $\begin{array}{c}{\left[\boldsymbol{X}_{\boldsymbol{d}} \boldsymbol{Y}_{\boldsymbol{d}} \boldsymbol{Z}_{\boldsymbol{d}}\right]} \\
(\mathbf{m m})\end{array}$ & $\begin{array}{c}\text { Ori } \\
(\mathbf{r a d})\end{array}$ & $\begin{array}{c}M . \boldsymbol{e}^{-\mathbf{6}} \\
\left(\mathbf{A . m ^ { 2 } )}\right.\end{array}$ & $\begin{array}{c}{\left[\boldsymbol{X}_{\boldsymbol{d}} \boldsymbol{Y}_{\boldsymbol{d}} \boldsymbol{Z}_{\boldsymbol{d}}\right]} \\
(\mathbf{m m})\end{array}$ & $\begin{array}{c}\text { Ori } \\
(\mathbf{r a d})\end{array}$ \\
\hline 1 & 2.3 & $2.1 ;-2.1 ; 0$ & 0 & 2.3 & $0 ; 0 ; 0$ & 0 \\
\hline 2 & 4.5 & $-6.3 ;-6.3 ; 0$ & 0 & 4.3 & $-6 ;-6 ; 0$ & 0 \\
\hline 3 & 6.9 & $-6.3 ; 6.3 ; 0$ & 0 & 4.9 & $-4.5 ; 7.5 ; 0$ & 0 \\
\hline 4 & 5 & $6.3 ; 2.1 ; 0$ & 0 & 4 & $-7.5 ; 3 ; 0$ & 0 \\
\hline 5 & 2.5 & $-2.1 ; 6.3 ; 0$ & 0 & 2.8 & $-1.5 ; 7.5 ; 0$ & 0 \\
\hline
\end{tabular}

Fig.11. shows the focusing magnitude as function of the virtual network size. The focusing amplitude is scaled by its maximum value. We observe that focusing deteriorates when the potential sources network size increases. If we increase the refining this mesh, we can obtain better results (accurate estimated behaviour), whereas treating the whole domain of calculation in this case could be time consuming.

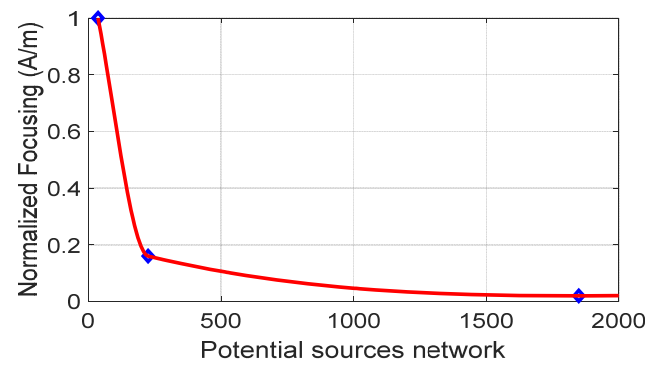

Fig. 11. Influence of potential sources network on focusing.

\section{Probe Radius}

The probe characteristics, in particular its radius, affect the accuracy of measured magnetic field. Indeed, when the probe has a weak radius, we have to increase the number of scanning points in order to measure correctly the spatial distribution of the field above the DUT. In this case, measurements errors are also increased, therefore the relative error on the obtained equivalent model. Yet, a large radius of the measuring probe means that the radiated field captured throughout the loop is no longer constant. In fact, the magnetic field is obtained by integrating the measured voltage at terminals of the probe using the following formula: $\mathrm{H}(t)=-\frac{1}{\mu_{0} \times S} \int_{0}^{t} \mathrm{~V}(t) d t$, where $\mu_{0}=$ $4 \times \pi \times 10^{-7} \mathrm{~N} / \mathrm{A}^{2}$ is the permeability in the free space and $S=\pi \times r^{2}$ is the surface of the measuring probe. Hence, possible measurement errors can add up with the field value calculated at the centre of the probe from the measured voltage. Therefore, in order to obtain accurate measurement, the minimum scanning resolution has to be equals the radius loop.

\section{DISCUSSION}

In the NF region, the field properties are determined using the characteristics of radiating sources. The total measured radiated field is the sum of the contributions from each active source.One needs to handle carefully the different parameters while evaluating the measurements and obtained results. Indeed, there exists several precautions that provide a better reconstruction. Hence, using previous configurations, a good compromise, that guarantees the accuracy of the equivalent model and an acceptable computing time, is based on the choice of involved parameters. Indeed, an increase of the size of potential sources network (Subsection II.C) enhance the obtained identification results and it is only based on simulation. Although, it is true that an increase in the size of TRM necessarily improves the quality of the reconstruction (Subsection II.B), but it costly since more measurements are required.

In this study, several simulation tests have been carried out. An adequate equivalent radiation model has been obtained using the EM inverse method based on EMTR technique. However, in 
order to enhance these results, of course an increase of the potential sources network dimensions improves the spatial focusing. Moreover, the accuracy of the method deteriorates when the size of this network is inferior to the size of TRM. Similar observations have been made while increasing the horizontal distance between the source and the scanning area. Yet, in the different tests, we obtain an accurate identification of the radiating sources locations and the estimation of radiated magnetic field maps is sufficient. Therefore, previous investigations have shown that the EM inverse method based on EMTR technique is efficient. It guarantees the convergence of the method and the uniqueness of the solution, since it is a matrix method.

\section{CONCLUSION}

In this paper, a parametric study has been carried out for the characterization of radiating sources in case of power electronic systems using the near field scanning technique. EM inverse method based on EMTR technique has been implemented using analytical equations of magnetic dipoles radiation. The proposed method has been presented using the time domain analysis. Indeed, this paper has presented the impact of parameters involved in the identification of a representative radiation model, such as the height of measurement, the TRM network, potential sources network, and the measuring loop radius. It is shown that a compromise between the accuracy of the obtained equivalent model and an acceptable computing time is provided using a high spatial resolution of both TRM and potential sources networks. Moreover, in order to enhance obtained results, we have to set adequately the scanning area in regard to the height of measurement.

Nevertheless, while taking experimental measurement, there exists other parameters that affect radiating sources characterization. Indeed, measurement errors such as coupling effects between the probe and the DUT, or the uncertainty of the measuring bench (spatial positioning of the probe, the antenna factor, etc.), highly affect the reconstruction quality and have to be minimized.

\section{ACKNOWLEDGMENT}

We thank the Ministry of Higher Education and Scientific Research of Tunisia, along with the Paris-Sud University for funding the international joint supervision of thesis (Cotutelle).

\section{REFERENCES}

[1] Paul, Clayton R. Introduction to electromagnetic compatibility. Vol. 184. John Wiley \& Sons, 2006.

[2] L. Beghou, F. Costa and L. Pichon, "Detection of Electromagnetic Radiations Sources at the Switching Time Scale Using an Inverse Problem-Based Resolution Method-Application to Power Electronic Circuits," in IEEE Transactions on Electromagnetic Compatibility, vol. 57, no. 1, pp. 52-60, Feb. 2015.

[3] S. Saidi and J. Ben Hadj Slama, "A Near-Field Technique Based on PZMI, GA, and ANN: Application to Power Electronics Systems," in IEEE Transactions on Electromagnetic Compatibility, vol. 56, no. 4, pp. 784-791, Aug. 2014

[4] Ravelo, Blaise, and Yang Liu. "Computation of transient near-field radiated by electronic devices from frequency data." Fourier Transform Applications. InTech, 2012.

[5] B. Zitouna and J. Ben Hadj Slama, "Enhancement of Time-Domain Electromagnetic Inverse Method for Modeling Circuits Radiations," in IEEE Transactions on Electromagnetic Compatibility, vol. 58, no. 2, pp. 534-542, April 2016

[6] S. Hedia, B. Zitouna, J. Ben Hadj Slama and L. Pichon, "Electromagnetic Time Reversal for Radiating Source Identification in Time Domain," 2018 15th International Multi-Conference on Systems, Signals \& Devices (SSD), Yassmine Hammamet, Tunisia, 2018, pp. 531-536.

[7] El Baba, I., Lalléchère, S., \& Bonnet, P. (2012). Time Reversal for Electromagnetism: Applications in Electromagnetic Compatibility. Trends in Electromagnetism: From Fundamentals to Applications, 177.

[8] S. Hedia, B. Zitouna, J. Ben Hadj Slama and L. Pichon, "A full time domain methodology based on near field time reversal for equivalent source identification," 2018 IEEE International Symposium on Electromagnetic Compatibility and 2018 IEEE Asia-Pacific Symposium on Electromagnetic Compatibility (EMC/APEMC), Singapore, 2018, pp. 141-146.

[9] S. Hedia, B. Zitouna, J. B. Hadj Slama and L. Pichon, "Comparative study between EMTR technique and a GA-based method for modeling EM radiation source in the Near Field," 2019 International Symposium on Electromagnetic Compatibility - EMC EUROPE, Barcelona, Spain, 2019, pp. 25-30.

[10] M. Fink, "Time reversal of ultrasonic fields. I. Basic principles," in IEEE Transactions on Ultrasonics, Ferroelectrics, and Frequency Control, vol 39, no. 5, pp. 555-566, Sept. 1992.

[11] F. Rachidi, M. Rubinstein and M. Paolone, Electromagnetic Time Reversal: Application to Electromagnetic Compatibility and Power Systems, Wiley, 2017

[12] G. Lugrin, N. M. Mora, F. Rachidi, M. Rubinstein and G. Diendorfer, "On the Location of Lightning Discharges Using Time Reversal of Electromagnetic Fields," in IEEE Transactions on Electromagnetic Compatibility, vol. 56, no. 1, pp. 149-158, Feb. 2014.

[13] Balanis, Constantine A. "Antenna Theory: Analysis \& Design, John Willey \& Sons." Inc. Publication (1997). 